\title{
Undergraduate students' understanding of the Moon phase change in a course for trainee teachers in compulsory education
}

\author{
Norihito Kawamura ${ }^{1, *}$ and Yuusei Naganuma ${ }^{2}$ \\ ${ }^{1}$ Akita University, Faculty of Education and Human Studies, 1-1 Tegatagakuen, Akita, Japan \\ ${ }^{2}$ Ochi Junior High School, 651 Ochi, Midori Ward, Chiba, Japan
}

\begin{abstract}
The authors conducted two classes for undergraduate students. The aim of these experimental classes is to find better way to reduce the number of students with misconceptions related to the Moon's phase change and increase the number of students with correct understanding of a lunar eclipse. To make deep knowledge of a lunar eclipse, in one class, we carried out practical work utilizing the scale size model kits. In another class, the students performed the work utilizing conceptual model kits. Finally, we found that the scale size model kit could reduce the number of students who believe the Moon phase change occurs due to a lunar eclipse.
\end{abstract}

\section{Introduction}

It is difficult to make students understand the phases of the moon. A decades-old issue as to study of the Moon has been discussed among Japanese educators and researchers. For instance, some researchers found that some people didn't understand the Moon phase change even though they were undergraduate students [1-3]. According to a recent research study, the most erroneous answer to the cause of the lunar phase change was a lunar eclipse [3]. In 2008, MEXT (Ministry of Education, Culture, Sports, Science and Technology, Japan) publicly released a new "Course of Study" as the national curriculum of Japan, which contains the study of the Moon in science classes at elementary and lower secondary school. As of 2017, undergraduate students aged 19 studied the Moon in science class only in lower secondary school under the former curriculum. Such students may have lower achievement on understanding the Moon due to fewer chances to study the Moon. Then, we attempt to reveal the actual situation of recent undergraduate students' cognition of lunar phase changes so that we may be able to develop an instructional method for improvement of understanding. Though some results of this study were already published in Japan [4], we show some results of the educational practices to improve the students' cognition.

\section{Analysis of contents for building up knowledge of the Moon phase change}

\footnotetext{
* Corresponding author: norihito@ed.akita-u.ac.jp
} 


\subsection{Objective of analysis}

To gain perspective for this study, we list basic knowledge and skills for understanding the lunar phase change, before reviewing results of the research. As of 2017, in Japan, the contents of study of the Moon in science class are conducted in 6th grade of elementary school and 3rd grade of lower secondary school.

\subsection{Basic knowledge related to contents in 6th grade of elementary school curriculum}

\subsubsection{Understanding of day and night hemispheres of the Moon}

The recent 'Course of Study' by MEXT states that 6th grade students should study the lunar phase change according to the changing positions of the Moon and Sun relative to the Earth [5]. Under this curriculum, the students also learn that the Moon has illuminated and dark hemispheres. Actually, science text books schematically illustrate sunlight, the Earth and the Moon on orbit looking down on Earth from the north pole of the ecliptic [6]. So the teachers may expect their students to understand that the Moon phase depends on the Moon's position in orbit around the Earth.

\subsection{Basic knowledge related to content in 3rd grade of lower secondary school curriculum}

\subsubsection{Concept of orbiting around the Earth}

According to "Course of Study" for lower secondary school [7], content science class deals with the period of the lunar phase change and the Moon's orbit around the Earth. For students, these concepts are very important to understand the Moon phase change.

\subsubsection{Moving of viewpoint from space to the Earth}

Utilizing schematic diagram showing the Moon's position in orbit around the Earth, we can infer the shape of the illuminating Moon as seen from the Earth. In that study, the students must mentally switch the observer's viewpoint from space to the ground. To improve the ability of changing the viewpoint is one of the issues in science class, because the performance is hard to improve for the students [8].

\subsubsection{Knowing difference between scale and schematic models}

Students frequently have the wrong notion that a not-to-scale schematic diagrams was actually drown to scale. A diagram showing the position relation of the Moon looking down on the Earth from the north pole of the ecliptic, is not illustrated to scale so that it may cause students' confusing the scale of diameter of astronomical bodies and the distance between them. When the teacher talks about the Moon phase change using such schematic figures, they should be careful to explain that not to scale models are shown in diagrams to prevent having misunderstanding.

\section{Questionnaire research}

\subsection{Objective of the research}


To study the situation of cognition by undergraduate students as preservice teachers, the authors conducted a series of questionnaire studies in SY (school year) 2015 and SY2016. We can get clues about how to improve young teachers' cognition about the Moon based on the results of research.

\subsection{Questionnaire targets}

The questionnaire surveyed to the undergraduate students who took the science education classes for elementary school teacher training course of Akita University in SY2015 and SY2016. A group of surveys consists of five questionnaires during a couples of months. The numbers of respondents are 83 (17 freshmen and 66 sophomores) in SY2015 and 88 (76 freshmen, 11 sophomores, and 1 junior student) in SY2016. In total, we gathered questionnaire sheets from 171 students.

\subsection{Question items}

The questionnaire sheets consist of five papers (Sheet 1 to 5). Outline of question items and response method on the sheets are as follows;

Sheet 1: Explanation of the lunar phase change (free description)

Sheet 2: Explanation of the Moon eclipse (free description)

Sheet 3: Drawing shadow on the Moon from both space and the Earth (drawing)

Sheet 4: Drawing shadow on the Moon based on the lunar phase diagram from space viewpoint (drawing)

Sheet 5: Experience of observation of the Moon and practice activity utilizing scale model kit of the Earth and the Moon (multiple choice)

Cross tabulation between results of Sheets 1 and 2 will reveal whether misconceptions are independent or not. Ability on changing viewpoint from space to the ground are based on the responses by drawings on Sheets 3 and 4. As to Sheet 5, we analyse whether misunderstanding would be related to experience with observations and practical activity or not.

\subsection{Implementation of research}

Five surveys of Sheet 1 to 5 in SY2015 were performed in approximately every three weeks during November 30, 2015 to February 22, 2016. Questionnaire sheets were collected just after 5 minutes except Sheet 5. Time for response of Sheet 5 was not limited so that the sheet was collected as soon as respondents replied. Surveys in SY2016 was carried out during October 17 to November 21, 2016 in the same way.

\subsection{Results}

On the basis of the cross tabulation for Sheet 1 to 5 , we found the results described below. The undergraduate students' understandings of the Moon phase are as follows;

- they understand sun light shines on half of the Moon,

- they can explain how lunar eclipses occur,

- they may not recognize that a schematic diagram of the Moon's orbit is drawn as a concept model,

- they may not know the period of the Moon orbit, and

- model experiment of the lunar phase change would not improve their understanding. 
In short, inadequate study in science classes at elementary and/or junior high schools might cause less achievement in understanding of the Moon phase change.

\section{Views of instruction about the Moon phase change for undergraduate students}

To improve understanding of the lunar phase change for undergraduate students, astronomical contents for $6^{\text {th }}$ graders should be taught in science education class in a teacher training course. Which teaching plan is better? Based on results mentioned above about misconceptions, the authors developed a couple of lessons on basic astronomy and science education for the students. e.g.,

1) basic understanding of size of diameter and orbits of the Moon the Earth,

2) basic understanding of evolution of the Moon, and

3) cognition that schematic diagrams in textbooks are "Conceptual Model".

\section{Educational practices}

\subsection{Empirical study using the model kits of the Moon and the Earth}

\subsubsection{The model kits}

To gain deep knowledge of the lunar phase change, the authors prepared two types of kits,

"Conceptual Model Kit (CMK)" and "Scale Size Model Kit (SMK)". CMK consists of the Moon and the Earth models made of Styrofoam whose diameters are $10 \mathrm{~cm}$ and $5 \mathrm{~cm}$. These astronomical bodies model are not to scale. When students study using this kit, the kit lets them do whatever they want to set distance between these model bodies. SMK consists of bigger and smaller balls which are scale of 1 to 200,000,000. When the students use this model, the distance of these objects is set about 1.9 meters.

\subsubsection{Framework of the educational practices}

After the questionnaire analysis, the authors conducted two classes (Classes C and S) in SY 2017 for the undergraduate students. To make deep knowledge related to the lunar phase change, the students of Class C used CMK. To improve understanding of lunar eclipse, the students of Class S performed the work utilizing SMK. The aim of such class organization is to find a better way to reduce the number of students with misconceptions related to Moon phase change and lunar eclipse.

\subsection{Assessment of the educational practices}

After conducting the classes by one of the authors, the authors carried out Sheets 1 and 2 surveys as post-survey to compare to the pre-survey results of the students. In these surveys, the students replied on the basis of knowledge of the Moon phase and lunar eclipse. The authors divided their replies into right and wrong answers. Fisher's exact test was used to compare the proportion of students having misconceptions about lunar eclipse and the Moon phase in the pre-survey with post-survey and was found to be significantly lower $(p=0.0085)$ in Class S. Finally, we found that SMK can reduce the number of students who believe the Moon phase change occurs due to lunar eclipse. 


\section{Discussion}

CMK did not improve the students' understanding of lunar phases because changing the viewpoints is hard for them and they do not have the experience of switching from different frames of reference. Why is changing viewpoints difficult for them? Witkin et al. identified field-dependence-independence dimension as a cognitive style [9]. Recently, the cognitive style has been applied with research on problems of science education. We may use proper teaching methods depending on whether the students' cognitive style are field-dependence or field-independence.

\section{Acknowledgements}

We thank our editor for improving the use of English in the manuscript.

\section{References}

1. R. Miyawaki, S. Nanbu, Earth Sci. (Chigaku Kyoiku), 45, 219 (1992)

2. A. Ito, M. Chisa, H. Tahara, Bull. Int. Res. Cent. Educ. Prac., Utsunomiya Univ., 30, 473 (2007)

3. T. Yunoki, Jour. Hokkaido Univ. Educ., 64, 151 (2014)

4. Y. Naganuma, N. Kawamura, Bull. Cent. Educ. Res. Prac., Fac. Edu. Hum. Stud., Akita Univ., 39, 81 (2017)

5. Ministry of Education, Culture, Sports, Science and Technology (Mext), Course of Study for elementary school, MEXT (2009)

6. S. Okamura, et. al., Modern Science 3 (revised edition), Tokyo Syoseki (2016)

7. Ministry of Education, Culture, Sports, Science and Technology (Mext), Course of Study for lower secondary school, MEXT (2009)

8. Y, Matsumori, Bull. Soc. Jap. Sci. Teac., 24, 27 (1983)

9. H. A. Witkin, et. al., Rev. Educ. Res., 47, 1(1975) 